should be confirmed in writing, particularly details of medicines. ${ }^{13}$ The reminder chart could help to satisfy these requirements. The labelling programmes used in general practice could easily be adapted to produce the reminder chart. Thus patients in hospital and general practice could be given this aid to compliance, which is effective, cheap, and not labour intensive.

We thank the Plain English Campaign for advice on the design of the sheet and Lorna Legg of Janssen Pharmaceuticals for advice on statistics. The research was funded by Yorkshire Regional Health Authority.

1 Parkin DM, Henney CR, Quirk J, Crooks J. Deviation from prescribed drug treatment after discharge from hospital. $B M \mathcal{F}$ 1976;ii:686-8.

2 Macdonald ET, Macdonald JB, Phoenix M. Improving compliance afte hospital discharge، $B M \mathcal{F} 1977$;ii:618-21.
3 Raynor DK. Patient compliance: the pharmacist's role. International foumal of Pharmacy Practice 1992;1:126-35.

4 Leeds pharmacist develops information sheet. Pharmaceutical fournal 1988 240:252

5 Raynor DK. Patient information and its influence on medication compliance. Bradford: University of Bradford, 1991. (PhD thesis.)

6 Gardner MJ, Gardner SB, Winter PD. Confidence interval analysis [computer program]. London: BMJ, 1989

7 Sweeney SJ, Dixon JS, Sutcliffe I. The impact of the clinical pharmacist on compliance in a geriatric population. Pharm f 1989;242:R4-6.

8 Sandler DA, Mitchell JRA, Fellows A, Garner ST. Is an information booklet for patients leaving hospital helpful and useful? $B M 7$ 1989;298:870-4.

9 Sandler DA, Heaton C, Gamer ST, Mitchell JRA. Patients' and general practitioners' satisfaction with inform praction Ellis DA, Hopkin JM, Leich AG, Crofon J. "Doctor's orders": cC the

11 Hallworth RB, Goldberg LA. Geriatric patients' understanding of labelling of medicines. Br F Pharm Pract 1984;6:42-8.

12 Mazzuca SA. Does patient education in chronic disease have therapeutic value? Fournal of Chronic Diseases 1982;35:521-9.

13 Department of Health. Discharge of patients from hospital. London: DoH, 1989. (HC(89)5.)

\title{
Abnormalities of sleep in patients with the chronic fatigue syndrome
}

\author{
Richard Morriss, Michael Sharpe, Ann L Sharpley, Philip J Cowen, Keith Hawton, Julie Morris
}

\section{MRC Clinical \\ Pharmacology Unit, Littlemore Hospital, Oxford OX4 4XN Richard Morriss, senior registrar in psychiatry Ann L Sharpley, fanssen research fellow \\ Philip J Cowen, clinical scientist}

University Department of Psychiatry, Warneford Hospital, Oxford OX3 7JX Michael Sharpe, clinical tutor in psychiatry

Keith Hawton, consultant psychiatrist

\section{Department of Medical Statistics, University Hospital of South Manchester, Manchester M20 8LR \\ Julie Morris, medical statistician}

\section{Correspondence to:} Dr R Morriss, Mental Illness Research Unit, Withington Hospital, Manchester M20 8LR.

\begin{abstract}
Objective-To determine whether patients with the chronic fatigue syndrome have abnormalities of sleep which may contribute to daytime fatigue.

Design-A case-control study of the sleep of patients with the chronic fatigue syndrome and that of healthy volunteers.
\end{abstract}

Setting-An infectious disease outpatient clinic and subjects' homes.

Subjects-12 patients who met research criteria for the chronic fatigue syndrome but not for major depressive disorder and 12 healthy controls matched for age, sex, and weight.

Main outcome measures-Subjective reports of sleep from patients' diaries and measurement of sleep patterns by polysomnography. Subjects' anxiety, depression, and functional impairment were assessed by interview.

Results-Patients with the chronic fatigue syndrome spent more time in bed than controls $(544 \mathrm{~min}$ $v 465 \mathrm{~min}, \mathrm{p}<0.001)$ but slept less efficiently $(90 \% v$ $96 \%, p<0.05)$ and spent more time awake after initially going to sleep $(31.9 \mathrm{~min} v 16.6 \mathrm{~min}, \mathrm{p}<0.05)$. Seven patients with the chronic fatigue syndrome had a sleep disorder (four had difficulty maintaining sleep, one had difficulty getting to sleep, one had difficulty in both initiating and maintaining sleep, and one had hypersomnia) compared with none of the controls $(p=0.003)$. Those with sleep disorders showed greater functional impairment than the remaining five patients (score on general health survey $50.4 \% v 70 \cdot 4 \%, p<0.05$ ), but their psychiatric scores were not significantly different.

Conclusions-Most patients with the chronic fatigue syndrome had sleep disorders, which are likely to contribute to daytime fatigue. Sleep disorders may be important in the aetiology of the syndrome.

\section{Introduction}

The chronic fatigue syndrome has been defined as fatigue that is medically unexplained, has lasted at least six months, and is associated with impaired physical and mental functioning. ${ }^{1}$ One of the commonest symptoms of the syndrome is subjective impairment of sleep. ${ }^{2}$ That this might be important in the syndrome's aetiology is suggested by the finding that experimental manipulation of sleep leads to increased fatigue. ${ }^{3}$ There have not, however, been any controlled studies of sleep patterns in patients with the chronic fatigue syndrome.

Many patients with the chronic fatigue syndrome also meet the criteria for major depressive disorder. ${ }^{4}$ About $60-70 \%$ of outpatients with major depressive disorder have a specific sleep abnormality of a shortened interval from the start of sleep to rapid eye movement sleep. ${ }^{5}$ It is therefore important to study the sleep of patients with the chronic fatigue syndrome who do not also have major depressive disorder. We have conducted a case-control study of such patients, using both subjective and objective measures of sleep.

\section{Subjects and methods}

We recruited the first 12 patients from the infectious disease outpatient clinic of a teaching hospital who met the following criteria: the patient's principal complaint was of subjective fatigue; the fatigue had been present at least half of the time for at least six months; fatigue was not a lifelong complaint; physical and mental functioning were both affected, with at least two types of activity (out of housework, recreation, social activity, occupation or studies, and independent living) being substantially impaired; the examining physician could find no evidence of physical disease; and, on a standardised psychiatric examination, ${ }^{6}$ the patient did not meet criteria for major depressive disorder. ${ }^{7}$ Thus the patients were not selected because of reported problems with sleeping. The patients were individually matched for age (within five years), sex, and weight (within $10 \mathrm{~kg}$ ) with healthy controls recruited from hospital staff.

Exclusion criteria for both patients and controls were the use of psychotropic drugs in the preceding three months, intake of more than six units of alcohol four times a week in the preceding three months, and a body mass index (weight $(\mathrm{kg}) /(\text { height }(\mathrm{m}))^{2}$ ) of $<18$ or $>25$. All subjects gave their informed consent to participate in the study, which was approved by the local psychiatric ethics committee.

Subjective assessment of sleep-The subjects kept a 24 hour diary of their sleep covering nine nights, including the two nights that polysomnograms were recorded. They recorded when they switched off their lights to go to sleep; the estimated time when they fell asleep; how often they woke in the night; the time of final awakening; the total time spent in bed; the quality of sleep (rated on a $10 \mathrm{~cm}$ visual analogue scale from 
worst ever (0) to best ever (100)); and how they felt in the morning in terms of mood, sleepiness, pain, weakness, and stiffness. They also recorded whether they had been disturbed by the recording equipment on the two nights of recording (rated on a five point scale of severity from not at all (0) to extremely (4)).

Objective assessment of sleep-Sleep montage electrodes were attached to the subjects and checked in the laboratory. The subjects then returned home to sleep in their own beds. Sleep records were obtained with a cassette recording system (Medilog 9000-11, Oxford Medical). The electroencephalogram was recorded from $\mathrm{C}_{4}-\mathrm{A}_{1}$ and $\mathrm{C}_{3}-\mathrm{A}_{2}$ sites. An electrooculogram and a submental electromyogram were also recorded. The sleep records were automatically analysed (Medilog sleep stager SS90111) and visually checked by an experienced sleep technician (ALS). The records were analysed to determine the percentage of time spent in $\alpha$ rhythm in sleep stages 2,3 , and 4 . If more than $25 \%$ of the time was spent in $\alpha$ rhythm we considered the patient to have the $\alpha$ non-rapid eye movement sleep abnormality found in fibromyalgia or fibrositis (a syndrome of muscle aches, muscle tenderness, and fatigue similar to chronic fatigue syndrome) ${ }^{89} \mathrm{We}$ also used the analysed records to detect the following sleep disorders: hypersomnia (if a patient's mean duration of sleep each night was more than two standard deviations above the mean for the control group); difficulty in initiating sleep (if a patient took more than one hour to get to sleep after turning out the lights); and difficulty in maintaining sleep (if a patient's mean time spent awake during the night was more than two standard deviations above the mean for the control group).

Clinical assessment-The subjects completed the brief symptom inventory, a measure of a range of psychiatric symptoms, ${ }^{10}$ and the hospital anxiety and

TABLE I-Functional impairment and psychiatric scores of patients with the chronic fatigue syndrome and controls

\begin{tabular}{|c|c|c|c|c|}
\hline & $\begin{array}{l}\text { Patients } \\
(n=12)\end{array}$ & $\begin{array}{l}\text { Controls } \\
(n=12)\end{array}$ & $\begin{array}{c}\text { Difference ( } 95 \% \\
\text { confidence interval) }\end{array}$ & $\mathrm{p}$ Value \\
\hline General health survey $(\%)^{\star}$ & $61 \cdot 3$ & 100 & $32.7(11.9$ to 66.8$)$ & $<0.01$ \\
\hline Brief symptom inventory & $31 \cdot 0$ & $5 \cdot 5$ & $27.0(9.0$ to 29.0$)$ & $<0.05$ \\
\hline \multicolumn{5}{|c|}{ Hospital anxiety and depression scale: } \\
\hline Depression $\dagger$ & $5 \cdot 4$ & 0.9 & $4 \cdot 5(2 \cdot 6$ to $6 \cdot 4)$ & $<0.001$ \\
\hline Anxietyt & $6 \cdot 1$ & $2 \cdot 8$ & $3.3(1.1$ to 5.5$)$ & $<0.01$ \\
\hline
\end{tabular}

*Median values compared by Wilcoxon matched pair rank sum test.

†Mean values compared by paired $t$ test.

TABLE II-Data on sleep reported by patients with the chronic fatigue syndrome and controls (values averaged over nine nights)

\begin{tabular}{lcccc}
\hline & $\begin{array}{c}\text { Patients } \\
(\mathrm{n}=12)\end{array}$ & $\begin{array}{c}\text { Controls } \\
(\mathrm{n}=12)\end{array}$ & $\begin{array}{c}\text { Difference }(95 \% \\
\text { confidence interval })\end{array}$ & $\mathrm{p}$ Value \\
\hline Time in bed (min) & 561 & 491 & $70(38$ to 101$)$ & $<0.001$ \\
Time asleep (min) $\dagger$ & 446 & 461 & $-14(-74$ to 45$)$ & NS \\
Time taken to fall asleep (min) $\dagger$ & 22.2 & 2.5 & $17.8(8.0$ to 42.0$)$ & $<0.05$ \\
No of periods awake† & 1.8 & 0.7 & $1.3(0.4$ to 1.9$)$ & $<0.01$ \\
\hline
\end{tabular}

* Mean values compared by paired $t$ test.

†Median values compared by Wilcoxon matched pair rank sum test.

TABLE III-Data (in minutes) from polysomnograms of patients with the chronic fatigue syndrome and controls (values averaged over two nights)

\begin{tabular}{|c|c|c|c|c|}
\hline & $\begin{array}{l}\text { Patients } \\
(n=12)\end{array}$ & $\begin{array}{l}\text { Controls } \\
(n=12)\end{array}$ & $\begin{array}{l}\text { Difference ( } 95 \% \\
\text { confidence interval) }\end{array}$ & $\mathrm{p}$ Value \\
\hline Time in bed ${ }^{\star}$ & 544 & 465 & $79(47$ to 111$)$ & $<0.001$ \\
\hline Time asleep ${ }^{\star}$ & 442 & 425 & $17(-25$ to 58$)$ & NS \\
\hline Sleep latencyt & $12 \cdot 2$ & $7 \cdot 8$ & $1 \cdot 2(-6$ to 16$)$ & NS \\
\hline Sleep efficiency $(\%)^{\star}$ & $90 \cdot 0$ & $95 \cdot 5$ & $-5 \cdot 4(-10$ to 1$)$ & $<0.05$ \\
\hline Time awake after start of sleep $\dagger$ & 31.9 & $16 \cdot 6$ & $18.5(-10$ to 56$)$ & $<0.05$ \\
\hline Stage 1 sleep $\star$ & $46 \cdot 7$ & $42 \cdot 3$ & $4 \cdot 4(-13$ to 21$)$ & NS \\
\hline Stage 2 sleep* & 214 & 190 & $24(-35$ to 59$)$ & NS \\
\hline Slow wave (stages 3 and 4 ) sleep* & 88.9 & $98 \cdot 8$ & $-9 \cdot 9(-34$ to 14$)$ & NS \\
\hline Rapid eye movement sleep ${ }^{\star}$ & $92 \cdot 4$ & 93.9 & $1.5(-11$ to 8$)$ & NS \\
\hline Rapid eye movement latencyt & 83.8 & $76 \cdot 0$ & $12 \cdot 8(-1$ to 29$)$ & NS \\
\hline$\alpha$ Rhythm in stages 2,3 , and 4 of sleep $(\%)^{\star}$ & $7 \cdot 1$ & $6 \cdot 0$ & $0.7(-5$ to 8$)$ & NS \\
\hline
\end{tabular}

${ }^{\star}$ Mean values compared by paired $t$ test.

†Median values compared by Wilcoxon matched pair rank sum test. depression scale." We examined the patients with the chronic fatigue syndrome for psychiatric disorders with the structured clinical interivew for Diagnostic and Statistical Manual of Mental Disorders Third Edition, Revised. ${ }^{6}$ We assessed the subjects' functional impairment with four subscales (physical, role, social functioning, and pain) of the short form general health survey of the medical outcome study. ${ }^{12}$ We recorded the score on each subscale as a percentage of the maximum and used the mean of the four scores as a measure of functional impairment. We also took a history of complaints of pain and fatigue and performed a physical examination to elicit tender points, looking at criteria diagnostic of fibromyalgia. ${ }^{13}$

Statistical analysis - We combined the data recorded in each subject's diary for the nine days of the study to get a mean daily score. We combined the two nights' polysomnographic data to get average scores because our method of recording at home does not require a night for adaptation, ${ }^{14}$ and initial analysis of our data showed that there was no significant difference between the data for the first and second nights for each of the groups. We compared cases and controls by means of the paired $t$ test for normally distributed variables and the Wilcoxon matched pairs rank sum test for other variables. The time spent awake recorded in the controls' polysomnograms had a log normal distribution, and we therefore constructed a reference range for the log data to determine which subjects had a sleep disorder (difficulty maintaining sleep). We examined the relation between polysomnographic data and other variables with Spearman rank correlations. We analysed the data with the SPSS program, apart from the confidence intervals, which we calculated manually. ${ }^{15}$

\section{Results}

Each group consisted of 10 women and two men. The patients had shown symptoms of fatigue for a median of 44.5 (range 17-100) months. The groups were similar in age (mean 36.1 (SD 8.7) years for patients and $35 \cdot 1(8 \cdot 3)$ years for controls) and weight $(61.9(4.2) \mathrm{kg}$ for patients and $60 \cdot 1(5.3) \mathrm{kg}$ for controls). One of the patients met research criteria for fibromyalgia. ${ }^{13}$ Table I shows the functional disability and psychiatric scores for the two groups. Six of the patients had a history of major depressive disorder. None of the controls had or had had a psychiatric disorder.

Compared with the controls, the patients reported spending significantly longer in bed at night, taking longer to fall asleep, and being awake more often during the night (table II). They also reported that they felt less refreshed on waking, lower in mood, and more sleepy; had more aches; and felt weaker and stiffer than the controls (all comparisons $\mathrm{p}<0.01$, data not shown). There were no significant differences between the patients and controls in the accuracy of their estimations of sleeping times. The recording of polysomnograms made no differences to the subjects' reports of their sleep patterns, and the CFS patients did not report being more disturbed by the recording equipment than the controls (data not shown)

The polysomnograms showed that the patients slept significantly less efficiently (total time asleep as a percentage of the period from the start of sleep to final waking) than the controls $(p<0.05)$ and were awake for significantly longer after the start of sleep $(p<0.05)$ (table III). The confidence intervals for the median values were slightly overestimated because of the small size of the groups and the method used to calculate the intervals. There were no significant differences between the CFS patients and controls in rapid eye movement latency; sleep latency (time taken to fall 


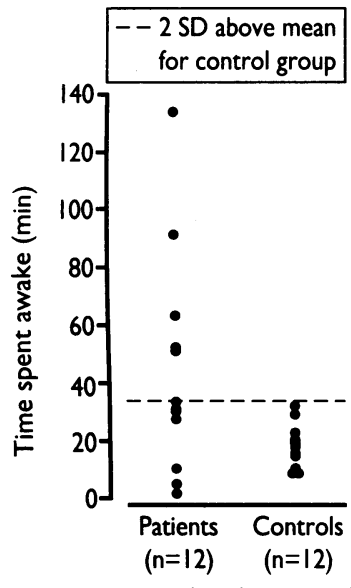

Time spent awake after start of sleep by patients with the chronic fatigue syndrome and controls asleep); time spent in any stage of sleep; or the percentage of $\alpha$ rhythm in stages 2, 3, and 4 of sleep (table III).

Seven of the patients met the criteria for a sleep disorder compared with none of the controls (Fisher's exact test, $p=0.003$ ). For five of these patients the disorder was an inability to maintain sleep. Examination of individual data showed that these five patients were responsible for the difference between the patients and controls in the median time spent awake after the start of sleep (figure). The remaining seven patients were similar to the controls in the time spent awake during the night. Two patients had difficulty getting to sleep, with sleep latencies of 71.3 and 83.5 minutes, and one had hypersomnia, sleeping for 103 minutes longer than the mean for the control group. One patient had difficulty in both initiating and maintaining sleep. Two patients (one of whom met clinical criteria for fibromyalgia) and two controls exhibited $\alpha$ non-rapid eye movement sleep abnormality.

The seven patients with sleep disorders showed significantly more functional impairment (general health survey score of $50 \cdot 4 \%$ (range $42 \cdot 5-71 \cdot 3 \%$ )) than the five other patients (general health survey score $70 \cdot 4 \%(49 \cdot 1-78 \cdot 3 \%)$ ) (Wilcoxon signed rank test, $T=$ $29, \mathrm{p}<0 \cdot 50$ ). Among the patients there was no significant correlation between psychiatric scores and any of the variables measured by polysomnography or between a history of major depression and any sleep abnormality.

\section{Discussion}

Although we studied only a small number of patients, they all met accepted criteria for the chronic fatigue syndrome, they were recruited from a single outpatient clinic, and their selection was not based on reported problems with sleeping. We therefore believe that they are likely to be representative of patients with the chronic fatigue syndrome who are referred to hospital. Their reports of their sleep correlated well with their electroencephalograms, indicating the validity of subjective reports of sleep by patients with this disorder.

Our findings suggest an association between sleep disorder and the chronic fatigue syndrome. Seven of the 12 subjects with the syndrome had a sleep disorder, five having difficulty maintaining sleep. Previous studies of sleep in patients with the syndrome either have not reported the start of sleep or its continuity (W B Mendelson et al. Milano International Symposium on sleep, hormones and immunological system. Milan, Italy) ${ }^{16}$ or were uncontrolled. ${ }^{17}$ Moldofsky reported that patients with postviral fatigue (similar to chronic fatigue syndrome) were awake more than twice as long as controls and took more than twice as long to fall asleep. ${ }^{18}$ The sample, however, was small and the findings were difficult to interpret because both the patients and the controls slept poorly in the sleep laboratory. The $\alpha$ non-rapid eye movement sleep abnormality described in patients with fibromyalgia ${ }^{9}$ has also been reported in patients with postviral fatigue. ${ }^{18}$ We were unable to replicate this finding, although the one patient in our study who met criteria for fibromyalgia also had the $\alpha$ non-rapid eye movement sleep abnormality.

We did not find the shortened rapid eye movement latency characteristic of major depressive disorder ${ }^{5}$ in the patients with the chronic fatigue syndrome. This is not surprising as we had excluded patients with diagnosable major depressive disorder, but our results are not consistent with the suggestion that the chronic fatigue syndrome is biologically identical with major depressive disorder but with a masking of the depres- sive symptoms in cases in which they are not clinically apparent. ${ }^{4}$

In keeping with most reported case series of patients with the syndrome, ${ }^{19}$ half of our patients reported a previous episode of major depressive disorder. We found no association, however, between a history of major depression and time spent in slow wave sleep, time spent awake after falling asleep, or the presence of a sleep disorder. None of the patients with a history of major depressive disorder and a current sleep disorder had decreased slow wave sleep (the abnormality thought to persist after major depressive disorder ${ }^{20}$ ) compared with the controls. Thus, although a history of major depressive disorder may explain sleep problems in some patients with the chronic fatigue syndrome, our findings do not support this suggestion.

Non-specific sleep abnormalities have been reported in patients suffering from minor emotional disorders such as anxiety ${ }^{21}$ or dysthymia. ${ }^{22}$ Although our patients had significantly more symptoms of anxiety and depression than the controls, there was no apparent relation between anxiety or depression and the presence of sleep abnormalities. To clarify this, future studies of the role of sleep in the chronic fatigue syndrome will need to control for not only major depressive disorder but also previous major depression and current level of emotional distress.

We suggest that sleep disorders may be important in the aetiology of the chronic fatigue syndrome, particularly since those patients with sleep disorder in our study tended to be the most functionally disabled. The subjective symptoms of feeling unrefreshed and physically weak, being sleepy, suffering poor concentration, and having muscle aches (similar to the symptoms of the chronic fatigue syndrome) can be induced in normal volunteers by a reduction or an increase in the duration of sleep of two hours each night over one week. ${ }^{23}$ The patients with sleep disorders in our study had an increase or decrease in their sleep time of about one to two hours each night compared with controls. The effects of sleep loss on performance are similar to the complaints of patients with the chronic fatigue syndrome: increased subjective effort on exertion $^{24}$ and slower subsequent recovery ${ }^{25}$ and reduced vigilance, attention span, and cognitive performance. $^{26}$

This work was supported by a grant from the University of Oxford Medical School. We thank Dr T Peto, consultant physician, John Radcliffe Hospital, Oxford, for allowing us to study his patients.

1 Sharpe MC, Archard LC, Banatvala JE, Borysiewicz LK, Clare AW, David A et al. A report-chronic fatigue syndrome: guidelines for research. $\mathcal{I} R$ Soc Med 1991;84:118-21.

2 Sharpe M, Hawton K, Seagroatt V, Pasvol G. Follow up of patients presenting with fatigue to an infectious diseases clinic. BMf 1992;305:147-52.

3 Horne JA. Dimensions to sleepiness. In: Monk TH, ed. Sleep, sleepiness and performance. Chichester: John Wiley, 1991:169-96.

4 Kendell RE. Chronic fatigue, viruses and depression. Lancet 1991;337:160-2.

5 Reynolds CF, Kupfer DJ. Sleep research in affective illness: state of the art circa 1987. Sleep 1987;10:199-215.

6 Spitzer RL, Williams JBW, Gibbon M, First MB. Structured clinical interview for DSM-111-R. Washington, DC: American Psychiatric Association, 1990.

7 American Psychiatric Association. Diagnostic and statistical manual of mental disorders, third edition, revised. Washington, DC: American Psychiatric Association, 1987

8 Moldofsky H, Scarisbrick P, England R, Smythe H. Musculoskeletal symptoms and non-REM sleep disturbance in patients with "fibrositis" syndrome and healthy subjects. Psychosom Med 1975;37:341-51.

9 Saskin P, Moldofsky H, Lue FA. Sleep and post-traumatic rheumatic pain modulation disorder (fibrositis syndrome). Psychosom Med 1986;48:319-23.

10 modulation disorder (fibrositis syndrome). Psychosom Med 1986;48:319-23. report. Psychol Med 1983;13:595-605.

11 Zigmond AS, Snaith RP. The hospital anxiety and depression scale. Acto Psychiatr Scand 1983;67:361-70.

12 Stewart AL, Hays RD, Ware JE. The MOS short-form general health survey. Med Care 1988;26:724-34.

13 Goldenberg DL. Fibromyalgia syndrome. JAMA 1987;257:2782-7.

14 Sharpley AL, Solomon RA, Cowen PJ. Evaluation of first night effect using ambulatory monitoring automatic sleep stage analysis. Sleep 1988;11:273-6. 5 Gardner MJ, Altman DG. Statistics with confidence. London: BMJ, 1989:9.

16 Whelton CL, Saskin P, Salit I, Moldofsky H. Postviral fatigue syndrome and sleep [abstract]. Sleep Research 1988;17:309. 
17 Krupp LB, Mendelson WB, Friedman R. An overview of chronic fatigue syndrome. F Clin Psychiatry 1991;52:403-10.

18 Moldofsky $\mathrm{H}$. Non-restorative sleep and symptoms after a febrile illness in patients with fibrositis and chronic fatigue syndromes. If Rheumatol 1989;16(suppl 19):150-3.

19 Abbey SE, Garfinkel PE. Chronic fatigue syndrome and the psychiatrist. Can I Psychiatry 1990;35:625-33.

20 Kupfer DJ. Maintenance treatment in recurrent depression: current and future directions. The first William Sargent lecture. $\mathrm{Br}$ F Psychiatry 1992;161:309-16.

21 Reynolds CF, Shaw DH, Newton T, Coble PA, Kupfer DJ. EEG sleep in outpatients with generalised anxiety: a preliminary comparison with depressed outpatients. Psychiatry Res 1983;8:81-9.
22 Arriga F, Rosado P, Paiva T. The sleep of dysthymic patients: a comparison with normal controls. Biol Psychiatry 1990;27:649-56.

23 Neyta N, Horne JA. Effects of sleep extension and reduction on mood in healthy adults. Human Psychopharmacology 1990;6:173-88.

24 Myles WS. Sleep deprivation, physical fatigue and the perception of exercise intensity. Med Sci Sports Exerc 1985;17:580-4.

25 McMurray RG, Brown CF. The effect of sleep loss on high intensity exercise and recovery. Aviat Space Environ Med 1984;55:1031-5.

26 Mackie RR. Vigilance, theory, operational performance and physiological correlates. New York: Plenum Press, 1977.

(Accepted 18 February 1993)

\title{
Coronary artery surgery: are women discriminated against?
}

\author{
Mark Petticrew, Martin McKee, Jeremy Jones
}

\section{Abstract}

Objective-To determine whether the sex differences in access to cardiac surgery observed in the United States exist in the United Kingdom.

Design-Retrospective analysis of routinely collected data.

Setting-South West Thames and North West Thames regional health authorities.

Subjects-8564 patients discharged from hospital with a principal diagnosis of coronary heart disease in 1987-8 in South West Thames region and 15243 discharges in North West Thames region in 1990-1.

Main outcome measures-Performance of angiography or coronary artery bypass surgery.

Results-In all age groups and among patients with a principal diagnosis of either angina or chronic ischaemia men were significantly more likely than women to undergo revascularisation in both regions. Using multiple logistic regression to control for potential clinical and demographic confounders, the male to female odds ratio for revascularisation among all cases was 1.59 (95\% confidence interval 1.25 to 2.03 ) in South West Thames region and 1.47 $(1.32$ to 1.63$)$ in North West Thames region.

Conclusion-There appears to be a systematic difference in the treatment received by men and women in the United Kingdom. The reasons for this are uncertain.

\section{Introduction}

There is a growing body of evidence that women in the United States are less likely than men to receive treatment for certain conditions. For chronic renal failure women are $20 \%$ less likely to be placed on dialysis, ${ }^{1}$ and, once on a dialysis programme, they are up to $50 \%$ less likely to receive a transplant. ${ }^{24}$ For coronary artery disease women with positive results on radionuclide exercise tests are one tenth as likely to be referred for surgery, ${ }^{5}$ those admitted for coronary artery disease are less likely to undergo angiography or revascularisation, ${ }^{6-8}$ and when women are referred for surgery it is at a later stage in the disease. ${ }^{9}$ Nevertheless, once a woman has undergone angiography she is almost as likely as a man to proceed to surgery. ${ }^{8}$ Healy has described this phenomenon as the Yentl syndrome, in which a woman can achieve equality only by being "just like a man." She argues that this can be done by, for example, showing that she has severe coronary artery disease on angiography. ${ }^{10}$

We cannot assume that the same results would be found in the United Kingdom because of the differences between the British and American health systems. For example, while the United Kingdom's National Health Service provides free care for all, many United States insurance plans have reduced the level of cover for dependants, thus relatively disadvantaging children and non-working women. ${ }^{11}$ There may also be as yet unidentified cultural differences. ${ }^{12}$

We have examined whether sex differences exist in gaining access to treatment for coronary heart disease in the United Kingdom. To ensure that any observed difference is not simply the consequence of decisions made in a single institution or by an individual doctor we used two large sets of hospital data.

\section{Methods}

Retrospective analyses were performed for patients discharged from hospitals in 13 districts in the South West Thames region during 1987-8 and for patients discharged in 13 districts in North West Thames region in 1990-1. The data available included the age, sex, primary diagnosis, up to four secondary diagnoses, and up to three procedure codes for each patient in South West Thames region. In North West Thames region up to six secondary diagnoses and up to four procedures were coded.

Patients with a primary diagnosis of either myocardial infarction (ICD-9 code 410), angina (code 413), chronic ischaemia (codes $414 \cdot 0-9$ ), or chest pain (code 786.5) were selected from the databases for further analysis. This produced two groups of patients with a primary diagnosis of coronary heart disease, numbering 8564 patients in South West Thames region and 15243 patients in North West Thames region.

For these patients the male to female odds of revascularisation by either angioplasty (Office of Population Censuses and Surveys (fourth revision) (OPCS 4) codes K49.1-K49.3, K49.8, K49.9, K50.1$\mathrm{K} 50.3$, K50.8, $\mathrm{K} 50.9$ ) or coronary artery bypass surgery (codes $\mathrm{K} 40.1-\mathrm{K} 40.9$ to $\mathrm{K} 48.1-\mathrm{K} 48.9$ ) were calculated.

The male to female odds ratios were stratified by primary diagnosis, by age (ages 30-49, 50-69, 70-89, and, in North West Thames, $\geqslant 90$ years), by secondary diagnosis of congestive heart failure (ICD- 9 codes 428 , $428.0,428.1,428.9$ ), and by secondary diagnosis of diabetes mellitus (ICD-9 codes 250, 250.0 to 250.7, 250.9 ) to control as far as possible variables confounding the relation between sex and rate of revascularisation. Sex differences in the distribution of these characteristics are shown in table I. $\chi^{2}$ Tests and the $\chi^{2}$ test for trend were used to calculate differences between men and women in the distributions of these characteristics.

We then calculated male to female odds ratios, using multiple logistic regression models to control for the potential confounders described above, with age entered as a continuous variable; $95 \%$ confidence intervals for each of these strata were also calculated. 